\title{
Symmetry Analysis for MHD Viscous Flow and Heat Transfer over a Stretching Sheet
}

\author{
Hossam S. Hassan \\ Department of Basic and Applied Science, Arab Academy for Science, Technology and Maritime Transport, \\ Alexandria, Egypt \\ Email: hossams@aast.edu
}

Received 26 October 2014; revised 22 November 2014; accepted 10 December 2014

Copyright (C) 2015 by author and Scientific Research Publishing Inc.

This work is licensed under the Creative Commons Attribution International License (CC BY).

http://creativecommons.org/licenses/by/4.0/

(c) (i) Open Access

\begin{abstract}
This work deals with the boundary layer flow and heat transfer of an electrically conducting viscous fluid over a stretching sheet. Lie-group method is applied for determining the symmetry reductions for the governing equations by reducing the number of independent variables in the given system of partial differential equations by one, leading to a system of non-linear ordinary differential equation. The resulting system is then solved numerically using shooting method coupled with Runge-Kutta scheme. Effects of various values of physical parameters on the horizontal and vertical velocities, temperature profiles, wall heat transfer and the wall shear stress (skin friction), have been studied and the results are plotted. Furthermore, a comparison between the present results with existing numerical and homotopy methods has been reported and we found that they are in a good agreement.
\end{abstract}

\section{Keywords}

MHD Flow, Viscous Flow, Stretching Sheet, Lie-Group, Similarity Solution

\section{Introduction}

The boundary layer flow and heat transfer of an incompressible viscous fluid over a stretching sheet appear in several manufacturing processes of industry such as the aerodynamic extrusion of plastic sheets, the extrusion of polymers, hot rolling, the cooling of metallic plates, glass-fiber production, etc., [1].

Sakiadis [2] presented the pioneering work in this field. He investigated the flow induced by a semi-infinite horizontally moving wall in an ambient fluid.

Crane [3] studied the flow over a linearly stretching sheet in an ambient fluid and gave a similarity solution in closed analytical form for the steady two-dimensional problem. He presented a closed form exponential solution 
for the planar viscous flow of linear stretching case.

Gupta and Gupta [4] investigated the effect of mass transfer on the Crane flow. They analyzed the viscous flow and heat transfer by an isothermal stretching sheet with suction/injection.

Chiam [5] studied the boundary layer flow due to a plate stretching with a power-low velocity distribution in presence of a magnetic field. To yield similarity equations, a special form of the magnetic field is chosen. He presented linearized solutions for the case of large magnetic parameters and derived an expression for the skin friction coefficient using Crocco's transformation and compared it numerically using Runge-Kutta shooting algorithm with Newton iteration.

Vajravelu [6] studied flow and heat transfer in a viscous fluid over a non-linear stretching sheet. In his study, the heat transfer is analyzed when the sheet is maintained at a constant temperature and the viscous dissipation is neglected. He used a fourth-order Runge-Kutta integration scheme to solve the resulting nonlinear differential equations.

Cortell [7] presented a numerical analysis for the flow and heat transfer in a viscous fluid over a nonlinear stretching sheet by employing a novel numerical procedure. In his work, he studied two cases for the nonlinear stretching sheet, with constant surface temperature and with prescribed surface temperature. The resulting nonlinear ordinary differential equations after converting the governing partial differential equations by a similarity transformation are solved using Runge-Kutta scheme.

Abbas and Hayat [8] studied the radiation effects on the magnetohydrodynamic (MHD) flow of an incompressible viscous fluid in a porous space. In their study, they extended the analysis of Cortell [7] by considering a MHD flow, analyzed the flow in a porous medium, included the radiation effects and provided analytic solution namely homotopy analysis method (HAM) instead of numerical technique applied in [7]. Hayat et al. [9] investigated the magnetohydrodynamic (MHD) boundary layer flow by employing the modified Adomian decomposition method and the Padé approximation and developed the series solution of the governing non-linear problem.

Ghotbi [10] considered the problem of the boundary layer flow of an incompressible viscous fluid over a nonlinear stretching sheet. In order to obtain analytical solution of the governing nonlinear differential equations, HAM is applied.

Mehmood et al. [11] reported the corrections to HAM results presented in [10]. A comparison between their HAM solution and the exact solution obtained by Pavlov [12] was made and it was in a good agreement.

Javed et al. [13] investigated the boundary layer flow and heat transfer analysis of electrically conducting viscous fluid over a nonlinearly shrinking sheet. They used a similarity transformation to reduce the governing partial differential equations to a set of nonlinear ordinary differential equations. The resulting system of equations is then solved numerically using an implicit finite difference scheme known as Keller-box method.

Fathizadeh et al. [14] employed the modification of the homotopy perturbation method to solve the MHD boundary-layer equations. In their work, the viscous fluid is electrically conducting in the presence of a uniform applied magnetic field and the induced magnetic field is neglected for small magnetic Reynolds number. They obtained the similarity solutions of ordinary differential equation resulting from the momentum equation. Some numerical comparisons among the new modified homotopy perturbation method, the standard homotopy perturbation, the exact solution and the shooting method are obtained.

In this paper, we shall investigate the solution of the MHD boundary layer flow for an incompressible viscous fluid over a sheet stretching according to a power-law velocity. Lie-group theory is applied to the equations of motion for determining symmetry reductions of partial differential equations [15]-[30]. The resulting system of nonlinear differential equations is then solved numerically using shooting method coupled with Runge-Kutta scheme. Our results are compared with the work of [5]-[14].

\section{Mathematical Formulation of the Problem}

We consider the MHD flow over a flat plate coinciding with the plane $\bar{y}=0$, of an incompressible viscous fluid with heat transfer. The wall is stretched horizontally by applying on both sides two equal and opposite forces along the $\bar{x}$-axis to keep the origin fixed. The fluid is electrically conducting under the influence of an applied magnetic field $B(\bar{x})$ in the $\bar{y}$-direction normally to the stretching sheet, Figure 1 .

The induced magnetic field is neglected. Under these assumptions, the continuity, momentum and energy equations become 


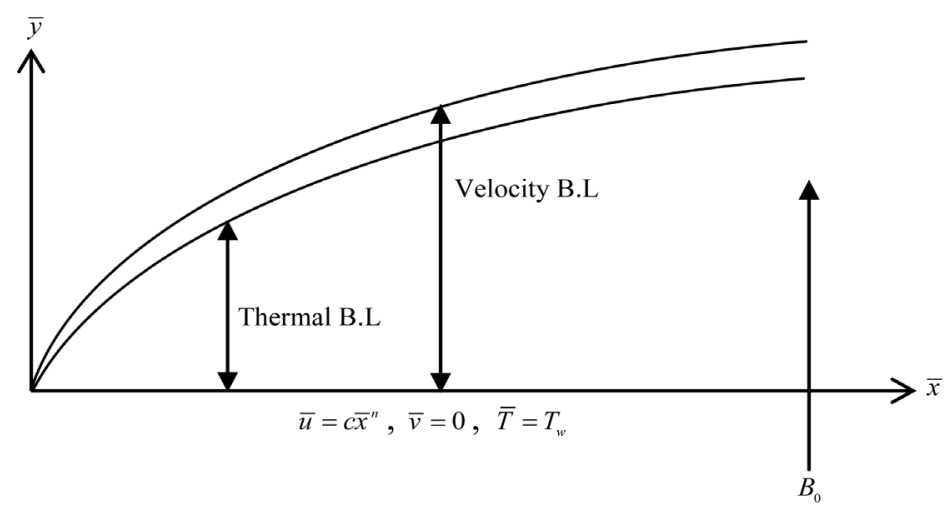

Figure 1. Physical model and coordinate system.

The continuity equation : $\frac{\partial \bar{u}}{\partial \bar{x}}+\frac{\partial \bar{v}}{\partial \bar{y}}=0$,

The momentum equation $: \bar{u} \frac{\partial \bar{u}}{\partial \bar{x}}+\bar{v} \frac{\partial \bar{u}}{\partial \bar{y}}=v \frac{\partial^{2} \bar{u}}{\partial \bar{y}^{2}}-\frac{\sigma B^{2}(\bar{x})}{\rho} \bar{u}$,

The energy equation : $\rho c_{p}\left(\bar{u} \frac{\partial \bar{T}}{\partial \bar{x}}+\bar{v} \frac{\partial \bar{T}}{\partial \bar{y}}\right)=\alpha \frac{\partial^{2} \bar{T}}{\partial \bar{y}^{2}}$,

where $\bar{u}$ and $\bar{v}$, are the velocity components in the $\bar{x}$ and $\bar{y}$ directions, respectively, $v$ is the kinematic viscosity, $\rho$ is the fluid density, $\sigma$ is the electrical conductivity of the fluid, $c_{p}$ is the specific heat of the fluid at constant pressure, $\alpha$ is the thermal conductivity of the fluid, and $\bar{T}$ is the temperature.

The magnetic field is defined by

$$
B(\bar{x})=B_{0} \bar{x}^{\frac{n-1}{2}},
$$

where $B_{0}$ and $n$ are constants.

The boundary conditions are

$$
\begin{aligned}
& \text { (i) } \bar{u}=c \bar{x}^{n}, \bar{v}=0, \bar{T}=T_{w} \text { at } \bar{y}=0, \\
& \text { (ii) } \bar{u} \rightarrow 0, \bar{T} \rightarrow T_{\infty} \text { as } \bar{y} \rightarrow \infty,
\end{aligned}
$$

where $c$ is a constant, $T_{w}$ is the uniform temperature of the stretching sheet and $T_{\infty}$ is the temperature at large distance from the wall, where $T_{w}>T_{\infty}$.

The variables in Equations (2.1)-(2.5) are dimensionless according to

$$
x=\frac{c \bar{x}}{U_{1}}, \quad y=\sqrt{\frac{(n+1) c}{2 v}} \bar{y}, \quad u=\frac{\bar{u}}{U_{1}}, \quad v=\sqrt{\frac{(n+1)}{2 c v}} \bar{v}, \quad T=\frac{\bar{T}-T_{\infty}}{T_{w}-T_{\infty}},
$$

where $U_{1}$ is the characteristic velocity.

Substitution from Equation (2.6) into Equations (2.1)-(2.3) gives

$$
\begin{aligned}
& \frac{\partial u}{\partial x}+\frac{\partial v}{\partial y}=0 \\
& u \frac{\partial u}{\partial x}+v \frac{\partial u}{\partial y}=\left(\frac{n+1}{2}\right) \frac{\partial^{2} u}{\partial y^{2}}-K x^{n-1} u, \\
& u \frac{\partial T}{\partial x}+v \frac{\partial T}{\partial y}=\left(\frac{n+1}{2}\right) \frac{1}{\operatorname{Pr}} \frac{\partial^{2} T}{\partial y^{2}},
\end{aligned}
$$


where, $K=\frac{\sigma B_{0}^{2}}{\rho c}$ is a constant, $\operatorname{Pr}=\frac{\mu c_{p}}{\alpha}$ is the Prandtl number, and $\mu=v \rho$ is the dynamic viscosity. Without losing of generality, let, $\left(\frac{U_{1}}{c}\right)^{n-1}=1$.

The boundary conditions Equation (2.5) will be

$$
\begin{aligned}
& \text { (i) } u=x^{n}, \quad v=0, T=1 \text { at } y=0, \\
& \text { (ii) } u \rightarrow 0, T \rightarrow 0 \text { as } y \rightarrow \infty .
\end{aligned}
$$

From the continuity Equation (2.7) there exist stream function $\Psi(x, y)$ such that,

$$
u(x, y)=\frac{\partial \Psi}{\partial y}, \quad v(x, y)=-\frac{\partial \Psi}{\partial x},
$$

which satisfies Equation (2.7) identically.

Substituting from Equation (2.11) into Equations (2.8)-(2.9), yields

$$
\begin{gathered}
\Psi_{y} \Psi_{x y}-\Psi_{x} \Psi_{y y}=\left(\frac{n+1}{2}\right) \Psi_{y y y}-K x^{n-1} \Psi_{y}, \\
\Psi_{y} T_{x}-\Psi_{x} T_{y}=\left(\frac{n+1}{2}\right) \frac{1}{\operatorname{Pr}} T_{y y},
\end{gathered}
$$

where subscripts denote partial derivatives.

The boundary conditions Equation (2.8) will be

$$
\begin{aligned}
& \text { (i) } \Psi_{y}=x^{n}, \Psi_{x}=0, T=1 \text { at } y=0, \\
& \text { (ii) } \Psi_{y} \rightarrow 0, T \rightarrow 0 \text { as } y \rightarrow \infty .
\end{aligned}
$$

\section{Solution of the Problem}

Firstly, we derive the similarity solutions using Lie-group method under which Equations (2.12)-(2.13) and the boundary conditions Equation (2.14) are invariant, and then we use these symmetries to determine the similarity variables.

Consider the one-parameter $(\varepsilon)$ Lie group of infinitesimal transformations in $(x, y ; \Psi, T)$ given by

$$
\begin{aligned}
& x^{*}=x+\varepsilon X(x, y ; \Psi, T)+O\left(\varepsilon^{2}\right), \\
& y^{*}=y+\varepsilon Y(x, y ; \Psi, T)+O\left(\varepsilon^{2}\right), \\
& \Psi^{*}=\Psi+\varepsilon \eta(x, y ; \Psi, T)+O\left(\varepsilon^{2}\right), \\
& T^{*}=T+\varepsilon \zeta(x, y ; \Psi, T)+O\left(\varepsilon^{2}\right),
\end{aligned}
$$

where " $\varepsilon$ " is the group parameter.

A system of partial differential Equations (2.12)-(2.13) is said to admit a symmetry generated by the vector field

$$
\Gamma \equiv X \frac{\partial}{\partial x}+Y \frac{\partial}{\partial y}+\eta \frac{\partial}{\partial \Psi}+\zeta \frac{\partial}{\partial T},
$$

if it is left invariant by the transformation $(x, y ; \Psi, T) \rightarrow\left(x^{*}, y^{*} ; \Psi^{*}, T^{*}\right)$.

The solutions $\Psi=\Psi(x, y)$ and $T=T(x, y)$, are invariant under the symmetry Equation (3.2) if

$$
\Phi_{\Psi}=\Gamma(\Psi-\Psi(x, y))=0, \text { when } \Psi=\Psi(x, y),
$$


and

$$
\Phi_{T}=\Gamma(T-T(x, y))=0 \text {, when } T=T(x, y) .
$$

Assume,

$$
\begin{aligned}
& \Delta_{1}=\Psi_{y} \Psi_{x y}-\Psi_{x} \Psi_{y y}-\left(\frac{n+1}{2}\right) \Psi_{y y y}+K x^{n-1} \Psi_{y}, \\
& \Delta_{2}=\Psi_{y} T_{x}-\Psi_{x} T_{y}-\left(\frac{n+1}{2}\right) \frac{1}{\operatorname{Pr}} T_{y y} .
\end{aligned}
$$

A vector $\Gamma$ given by Equation (3.2), is said to be a Lie point symmetry vector field for Equations (2.12)(2.13) if

$$
\left.\Gamma^{[3]}\left(\Delta_{j}\right)\right|_{\Delta_{j}=0}=0, \quad j=1,2,
$$

where,

$$
\begin{aligned}
\Gamma^{[3]} \equiv & X \frac{\partial}{\partial x}+Y \frac{\partial}{\partial y}+\eta \frac{\partial}{\partial \Psi}+\zeta \frac{\partial}{\partial T}+\eta^{x} \frac{\partial}{\partial \Psi_{x}}+\eta^{y} \frac{\partial}{\partial \Psi_{y}}+\zeta^{x} \frac{\partial}{\partial T_{x}}+\zeta^{y} \frac{\partial}{\partial T_{y}} \\
& +\eta^{x y} \frac{\partial}{\partial \Psi_{x y}}+\eta^{y y} \frac{\partial}{\partial \Psi_{y y}}+\zeta^{y y} \frac{\partial}{\partial T_{y y}}+\eta^{y y y} \frac{\partial}{\partial \Psi_{y y y}},
\end{aligned}
$$

is the third prolongation of $\Gamma$.

To calculate the prolongation of the given transformation, we need to differentiate Equation (3.1) with respect to each of the variables, $x$ and $y$. To do this, we introduce the following total derivatives

$$
\begin{aligned}
& D_{x} \equiv \partial_{x}+\Psi_{x} \partial_{\Psi}+T_{x} \partial_{T}+\Psi_{x x} \partial_{\Psi_{x}}+T_{x x} \partial_{T_{x}}+\Psi_{x y} \partial_{\Psi_{y}}+\cdots, \\
& D_{y} \equiv \partial_{y}+\Psi_{y} \partial_{\Psi}+T_{y} \partial_{T}+\Psi_{y y} \partial_{\Psi_{y}}+T_{y y} \partial_{T_{y}}+\Psi_{x y} \partial_{\Psi_{x}}+\cdots,
\end{aligned}
$$

Equation (3.7) gives the following linear partial differential equation

$$
\begin{aligned}
& (n-1) K X x^{n-2} \Psi_{y}-\eta^{x} \Psi_{y y}+\eta^{y}\left[\Psi_{x y}+K x^{n-1}\right]+\eta^{x y} \Psi_{y}-\eta^{y y} \Psi_{x}-\left(\frac{n+1}{2}\right) \eta^{y y y}=0, \\
& -\eta^{x} T_{y}+\eta^{y} T_{x}+\zeta^{x} \Psi_{y}-\zeta^{y} \Psi_{x}-\left(\frac{n+1}{2}\right) \frac{1}{\operatorname{Pr}} \zeta^{y y}=0 .
\end{aligned}
$$

The components $\eta^{x}, \eta^{y}, \zeta^{x}, \zeta^{y}, \eta^{x y}, \eta^{y y}, \zeta^{y y}, \eta^{y y y}$ can be determined from the following expressions

$$
\begin{aligned}
& \eta^{S}=D_{S} \eta-\Psi_{x} D_{S} X-\Psi_{y} D_{S} Y, \\
& \zeta^{S}=D_{S} \zeta-T_{x} D_{S} X-T_{y} D_{S} Y, \\
& \eta^{J S}=D_{S} \eta^{J}-\Psi_{J x} D_{S} \phi-\Psi_{J y} D_{S} \zeta, \\
& \zeta^{J S}=D_{S} \zeta^{J}-T_{J x} D_{S} X-T_{J y} D_{S} Y,
\end{aligned}
$$

where $S$ and $J$ are stand for $x$ and $y$.

Invariance of the boundary conditions Equation (2.14i), yields

$$
\zeta=0 \text {. }
$$

Substitution from Equations (3.12)-(3.13) into Equation (3.11) will lead to a large expression, then, equating to zero the coefficients of $T_{x y}, \Psi_{y} T_{x y}, T_{y} T_{x y}, \Psi_{y} T_{x} T_{y}, \Psi_{x} \Psi_{y} T_{y}$ and $T_{x}$, gives 


$$
X_{y}=X_{\Psi}=X_{T}=Y_{T}=Y_{\Psi}=\eta_{y}=0 .
$$

Substitution from Equation (3.14) into Equation (3.11) will remove many terms. Then, equating to zero the coefficients of $T_{y}$ and $\Psi_{x} T_{y}$, leads to the following system of determining equations:

$$
\begin{gathered}
\eta_{x}-\left(\frac{n+1}{2}\right) \frac{1}{\operatorname{Pr}} Y_{y y}=0, \\
X_{x}-Y_{y}-\eta_{\Psi}=0 .
\end{gathered}
$$

Again, substitution from Equations (3.12)-(3.16) into Equation (3.10) will remove many terms. Then, equating to zero the coefficients of $T_{y}, \Psi_{x} \Psi_{y}, \Psi_{y y},\left(\Psi_{y}\right)^{2}, \Psi_{x}\left(\Psi_{y}\right)^{2}$ and $\Psi_{y}$, gives

$$
\eta_{T}=Y_{y y}=\eta_{x}=Y_{x y}=\eta_{\Psi \Psi}=0 \text {, }
$$

and

$$
X-\left(\frac{2}{1-n}\right) x Y_{y}=0
$$

Solving the system of Equations (3.14)-(3.18) in view of the invariance of the boundary conditions Equation (2.14), yields

$$
X=\frac{2 c_{1} x}{1-n}, \quad Y=c_{1} y+c_{2}, \quad \eta=\left(\frac{1+n}{1-n}\right) c_{1} \Psi+c_{3}, \quad \zeta=0 .
$$

The system of nonlinear Equations (2.12)-(2.13) has the three-parameter Lie group of point symmetries generated by

$$
\Gamma_{1} \equiv \frac{2 x}{1-n} \frac{\partial}{\partial x}+y \frac{\partial}{\partial y}+\frac{1+n}{1-n} \Psi \frac{\partial}{\partial \Psi}, \quad \Gamma_{2} \equiv \frac{\partial}{\partial y} \quad \text { and } \quad \Gamma_{3} \equiv \frac{\partial}{\partial \Psi} .
$$

The one-parameter group generated by $\Gamma_{1}$ consists of scaling, whereas $\Gamma_{2}$ and $\Gamma_{3}$ consists of translation. The commutator table of the symmetries is given in Table 1 , where the entry in the $i$-th row and $j$-th column is defined as $\left[\Gamma_{i}, \Gamma_{j}\right]=\Gamma_{i} \Gamma_{j}-\Gamma_{j} \Gamma_{i}$.

The finite transformations corresponding to the symmetries $\Gamma_{1}, \Gamma_{2}$ and $\Gamma_{3}$ are respectively

$$
\left.\begin{array}{llll}
\Gamma_{1}: x^{*}=\mathrm{e}^{\frac{2}{1-n} \varepsilon_{1}} x, & y^{*}=\mathrm{e}^{\varepsilon_{1}} y, & \Psi^{*}=\mathrm{e}^{\frac{1+n}{1-n} \varepsilon_{1}} \Psi, & T^{*}=T \\
\Gamma_{2}: x^{*}=x, & y^{*}=y+\varepsilon_{2}, & \Psi^{*}=\Psi, & T^{*}=T \\
\Gamma_{3}: x^{*}=x, & y^{*}=y, & \Psi^{*}=\Psi+\varepsilon_{3}, & T^{*}=T
\end{array}\right\},
$$

where $\varepsilon_{1}, \varepsilon_{2}$ and $\varepsilon_{3}$ are the group parameters.

We look for solutions that invariant under the linear combination of the operators given by Equation (3.20). By determine the one-dimensional optimal system of subalgebras of the given partial differential equation, all of these solutions can be obtained. Olver's approach given in [17] starts out by computing the commutators of the

Table 1. Table of commutators of the basis operators.

\begin{tabular}{cccc}
\hline & $\Gamma_{1}$ & $\Gamma_{2}$ & $\Gamma_{3}$ \\
\hline$\Gamma_{1}$ & 0 & $-\Gamma_{2}$ & $-\frac{1+n}{1-n} \Gamma_{3}$ \\
$\Gamma_{2}$ & $\Gamma_{2}$ & 0 & 0 \\
$\Gamma_{3}$ & $\frac{1+n}{1-n} \Gamma_{3}$ & 0 & 0 \\
\hline
\end{tabular}


symmetry Lie algebra Equation (3.20) and then obtaining the adjoint representations. The adjoint action on Lie algebras is defined by the adjoint operator given by

$$
\operatorname{Ad}_{\exp \left(a \Gamma_{i}\right)}\left\langle\Gamma_{j}\right\rangle=\mathrm{e}^{-a \Gamma_{i}} \Gamma_{j} \mathrm{e}^{a \Gamma_{i}},
$$

where, $a$ is a small parameter.

In terms of Lie brackets using Campbell-Baker-Hausdorff theorem [31], this operator can be rewritten as

$$
\operatorname{Ad}_{\exp \left(a \Gamma_{i}\right)}\left\langle\Gamma_{j}\right\rangle=\Gamma_{j}-a\left[\Gamma_{i}, \Gamma_{j}\right]+\frac{a^{2}}{2 !}\left[\Gamma_{i},\left[\Gamma_{i}, \Gamma_{j}\right]\right]-\cdots .
$$

In our problem, $\Omega=\left\langle\Gamma_{1}, \Gamma_{2}, \Gamma_{3}\right\rangle$ is the Lie algebra associated with the symmetry group. The calculations of the adjoint action are summarized in Table 2.

To construct the one-dimensional optimal system of $\Omega$, consider a general element of $\Omega$ given by

$$
G=a_{1} \Gamma_{1}+a_{2} \Gamma_{2}+a_{3} \Gamma_{3},
$$

for some constants $a_{1}, a_{2}$ and $a_{3}$, and probe whether $G$ can be transformed to a new element $G^{\prime}$ under the general adjoint action, where $G^{\prime}$ takes a simpler form than $G$, [32].

Let,

$$
G^{\prime}=\operatorname{Ad}_{\exp \left(a \Gamma_{i}\right)}\langle G\rangle=a_{1}^{\prime} \Gamma_{1}+a_{2}^{\prime} \Gamma_{2}+a_{3}^{\prime} \Gamma_{3} .
$$

We make appropriate choice of $a$ such that the $a_{i}^{\prime}$ 's can be made 0 or 1 . We end up with simpler forms of $G$ that will constitute the one-dimensional optimal system.

By substitution $\Gamma_{i}=\Gamma_{2}$ in Equation (3.25) and dropping the primes, we get

$$
G^{\prime}=a_{1} \Gamma_{1}+\left(a_{2}-a a_{1}\right) \Gamma_{2}+a_{3} \Gamma_{3} .
$$

Now, Equation (3.26) prompts the consideration of the cases $a_{1} \neq 0$ and $a_{1}=0$.

Case (1): $a_{1} \neq 0$

By choosing $\left(a=a_{2} / a_{1}\right)$ and scaling the resulting operator by $a_{1}$, Equation (3.26) will be

$$
G^{\prime}=\Gamma_{1}+a_{3} \Gamma_{3} .
$$

We can further consider the subcases $a_{3} \neq 0$ and $a_{3}=0$. Therefore, an optimal system of one-dimensional subalgebra for this case is given by $\left\{\Gamma_{1}, \Gamma_{1}+\delta \Gamma_{3}\right\}$, where, $\delta \in R$.

Case (2): $a_{1}=0$

Using repeatedly the adjoint operation to simplify $G$, an optimal system of one-dimensional subalgebra for this case is given by $\left\{\Gamma_{2}, \Gamma_{2}+\gamma \Gamma_{3}\right\}$, where, $\gamma \in R$.

In summary, the optimal system of one-dimensional subalgebras of the symmetry Lie algebra is

$$
\Theta=\left\{\Gamma_{1}, \Gamma_{2}, \Gamma_{1}+\delta \Gamma_{3}, \Gamma_{2}+\gamma \Gamma_{3}\right\} .
$$

Table 3 shows the solution of the invariant surface conditions associated with the optimal system.

(i) Solutions invariant under $\Gamma_{1}$ :

The characteristic

Table 2. Table of adjoint representations.

\begin{tabular}{cccc}
\hline Ad & $\Gamma_{1}$ & $\Gamma_{2}$ & $\Gamma_{3}$ \\
\hline$\Gamma_{1}$ & $\Gamma_{1}$ & $\mathrm{e}^{a} \Gamma_{2}$ & $\mathrm{e}^{\frac{1+n}{1-n}} \Gamma_{3}$ \\
$\Gamma_{2}$ & $\Gamma_{1}-a \Gamma_{2}$ & $\Gamma_{2}$ & $\Gamma_{3}$ \\
$\Gamma_{3}$ & $\Gamma_{1}-\frac{1+n}{1-n} a \Gamma_{3}$ & $\Gamma_{2}$ & $\Gamma_{3}$ \\
\hline
\end{tabular}


Table 3. Solutions of the invariant surface conditions associated with the optimal system.

\begin{tabular}{ccc}
\hline Characteristic $\Phi=\left(\Phi_{\Psi}, \Phi_{T}\right)$ & Solutions of the invariant surface conditions \\
\hline$\Gamma_{1}$ & $\Phi_{\Psi}=\frac{1+n}{1-n} \Psi-\frac{2 x}{1-n} \Psi_{x}-y \Psi_{y}, \Phi_{T}=-\frac{2 x}{1-n} T_{x}-y T_{y}$ & $\Psi=x^{\frac{n+1}{2}} F(\lambda), T=\theta(\lambda), \lambda=y x^{\frac{n-1}{2}}$ \\
$\Gamma_{2}$ & $\Phi_{\Psi}=-\Psi_{y}, \Phi_{T}=-T_{y}$ & $\Psi=\Psi(x), T=T(x)$ \\
$\Gamma_{1}+\delta \Gamma_{3}$ & $\Phi_{\Psi}=\frac{1+n}{1-n} \Psi+\delta-\frac{2 x}{1-n} \Psi_{x}-y \Psi_{y}, \Phi_{T}=-\frac{2 x}{1-n} T_{x}-y T_{y}$ & $\Psi=x^{\frac{n+1}{2}} F(\lambda)-\frac{1-n}{1+n} \delta, T=\theta(\lambda), \lambda=y x^{\frac{n-1}{2}}$ \\
$\Gamma_{2}+\gamma \Gamma_{3}$ & $\Phi_{\Psi}=\gamma-\Psi_{y}, \Phi_{T}=-T_{y}$ & $\Psi=\gamma y+g(x), T=T(x)$ \\
\hline
\end{tabular}

$$
\Phi=\left(\Phi_{\Psi}, \Phi_{T}\right)
$$

has the components

$$
\Phi_{\Psi}=\frac{1+n}{1-n} \Psi-\frac{2 x}{1-n} \Psi_{x}-y \Psi_{y}, \quad \Phi_{T}=-\frac{2 x}{1-n} T_{x}-y T_{y} .
$$

Therefore, the general solutions of the invariant surface conditions Equations (3.3)-(3.4) are

$$
\Psi=x^{\frac{n+1}{2}} F(\lambda), \quad T=\theta(\lambda),
$$

where $\lambda=y x^{\frac{n-1}{2}}$ is the similarity variable.

Substitution from Equation (3.31) into Equations (2.12)-(2.13), yields

$$
\begin{aligned}
& \frac{\mathrm{d}^{3} F}{\mathrm{~d} \lambda^{3}}+F \frac{\mathrm{d}^{2} F}{\mathrm{~d} \lambda^{2}}-\beta\left(\frac{\mathrm{d} F}{\mathrm{~d} \lambda}\right)^{2}-M \frac{\mathrm{d} F}{\mathrm{~d} \lambda}=0, \\
& \frac{\mathrm{d}^{2} \theta}{\mathrm{d} \lambda^{2}}+\operatorname{Pr} F \frac{\mathrm{d} \theta}{\mathrm{d} \lambda}=0,
\end{aligned}
$$

where, $\beta=\frac{2 n}{n+1}$ and $M=\frac{2 K}{n+1}$ is the magnetic parameter, where $\sqrt{M}$ is the Hartmann number.

The boundary conditions Equation (2.14) will be

$$
\begin{aligned}
& \text { (i) } \frac{\mathrm{d} F}{\mathrm{~d} \lambda}=1, \quad F=0, \quad \theta=1 \text { at } \lambda=0, \\
& \text { (ii) } \frac{\mathrm{d} F}{\mathrm{~d} \lambda} \rightarrow 0, \quad \theta \rightarrow 0 \text { as } \lambda \rightarrow \infty .
\end{aligned}
$$

(ii) Solutions invariant under $\Gamma_{2}$ :

The characteristic Equation (3.29) has the components

$$
\Phi_{\Psi}=-\Psi_{y}, \quad \Phi_{T}=-T_{y} .
$$

Therefore, the general solutions of the invariant surface conditions Equations (3.3)-(3.4) are

$$
\Psi=\Psi(x), \quad T=T(x) .
$$

Practically, Equation (3.36) is a solution of Equations (2.12)-(2.13), even though it is not a particularly interesting one which contradicts the boundary conditions Equation (2.14). So, no solutions are invariant under the group generated by $\Gamma_{2}$.

(iii) Solutions invariant under $\Gamma_{1}+\delta \Gamma_{3}$ :

The characteristic Equation (3.29) has the components 


$$
\Phi_{\Psi}=\frac{1+n}{1-n} \Psi+\delta-\frac{2 x}{1-n} \Psi_{x}-y \Psi_{y}, \quad \Phi_{T}=-\frac{2 x}{1-n} T_{x}-y T_{y} .
$$

Therefore, the general solutions of the invariant surface conditions Equations (3.3)-(3.4) are

$$
\Psi=x^{\frac{n+1}{2}} F(\lambda)-\frac{1-n}{1+n} \delta, \quad T=\theta(\lambda),
$$

where $\lambda=y x^{\frac{n-1}{2}}$ is the similarity variable, which gives the same solutions invariant under $\Gamma_{1}$.

(iv) Solutions invariant under $\Gamma_{2}+\gamma \Gamma_{3}$ :

The characteristic Equation (3.29) has the components

$$
\Phi_{\Psi}=\gamma-\Psi_{y}, \quad \Phi_{T}=-T_{y} .
$$

Therefore, the general solutions of the invariant surface conditions Equations (3.3)-(3.4) are

$$
\Psi=\gamma y+g(x), \quad T=T(x) .
$$

This contradicts the boundary conditions Equation (2.14). So, no solutions are invariant under the group generated by $\Gamma_{2}+\gamma \Gamma_{3}$.

\section{Results and Discussion}

The system of non-linear differential Equations (3.32)-(3.33) with the boundary conditions Equation (3.34) is solved numerically using the shooting method, coupled with Runge-Kutta scheme. From Equations (2.11) and (3.31), we get

$$
U=\frac{u}{x^{n}}=\frac{\mathrm{d} F}{\mathrm{~d} \lambda}, \quad V=\frac{v}{x^{\frac{n-1}{2}}}=-\frac{n+1}{2}\left[F+\left(\frac{n-1}{n+1}\right) \lambda \frac{\mathrm{d} F}{\mathrm{~d} \lambda}\right] .
$$

The effects of the parameter $\beta$ which is a function of the power-index $n$, the Hartmann number $\sqrt{M}$, and the Prandtl number $\operatorname{Pr}$ on the horizontal and vertical velocities, and temperature profiles are illustrated in Figures 2-8. Moreover, the numerical values of the skin friction $f^{\prime \prime}(0)$ (wall shear stress) and rate of heat transfer $-\theta^{\prime}(0)$ are tabulated in Tables 4-11, for different values of parameters of interest.

\subsection{The Horizontal Velocity}

Figure 2 illustrates the effect of $\beta$ on the profile of the horizontal velocity $U$. It is noted that, the horizontal velocity decreases as $\beta$ increases both for $M=0$ (hydrodynamic fluid) and $M=1$ (hydromagnetic fluid) but this decreasing is smaller with $M=1$ compared with the case $M=0$, that is because the magnetic force acts as a resistance to the flow, [13]. Also, the boundary layer thickness decreases by increasing $\beta$ and the flow makes the stretching surface rougher.

Figure 3 describes the effect of $M$ on the behavior of the horizontal velocity $U$. As seen, by increasing the magnetic field, the horizontal velocity and the thickness of the boundary layer decrease. From Figure 3(a) we can conclude that, for $\beta=-1.5$ with small values of $M$ less than 0.4 near the surface, the behavior of the horizontal velocity is differ from the well-known cases, that is because the horizontal velocity increases to a maximum values before it starts to decrease.

\subsection{The Vertical Velocity}

Figure 4 shows the behaviour of the vertical velocity $V$ for $\beta=1.5$, over a range of the magnetic parameter $M$. As seen, the absolute value of the vertical velocity increases with the decrease of $M$.

Figure 5 illustrates the behaviour of the vertical velocity $V$ for $M=1$ over a range of the parameter $\beta$. As seen, the absolute value of the vertical velocity increases with the increase of $\beta$. 


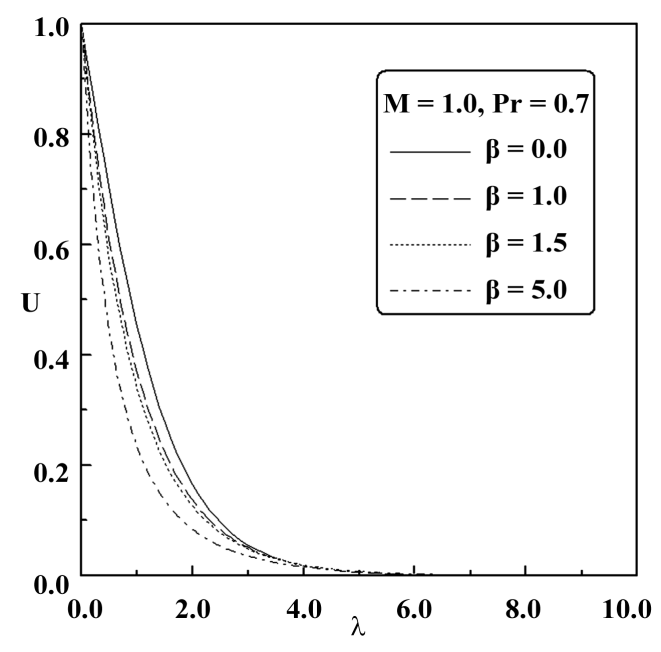

(a)

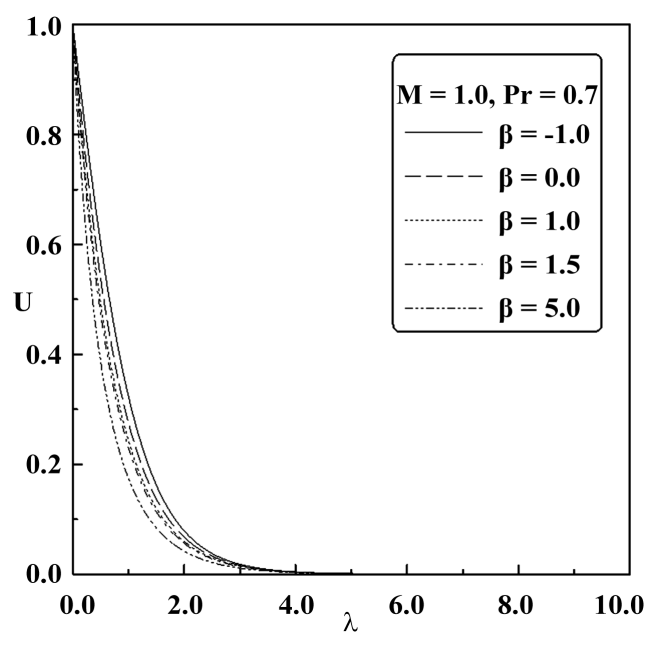

(b)

Figure 2. Horizontal velocity profiles over a range of $\beta$ with $\operatorname{Pr}=0.7$ for: (a) $M=0$; (b) $M=1$.

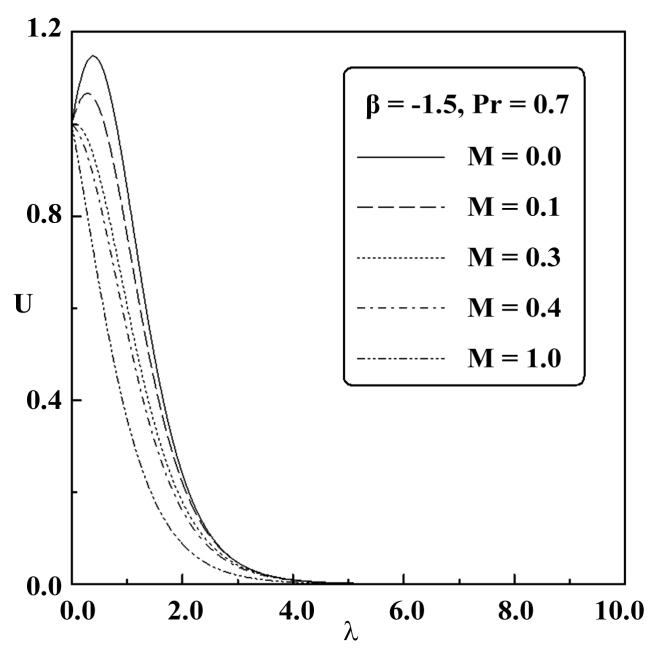

(a)

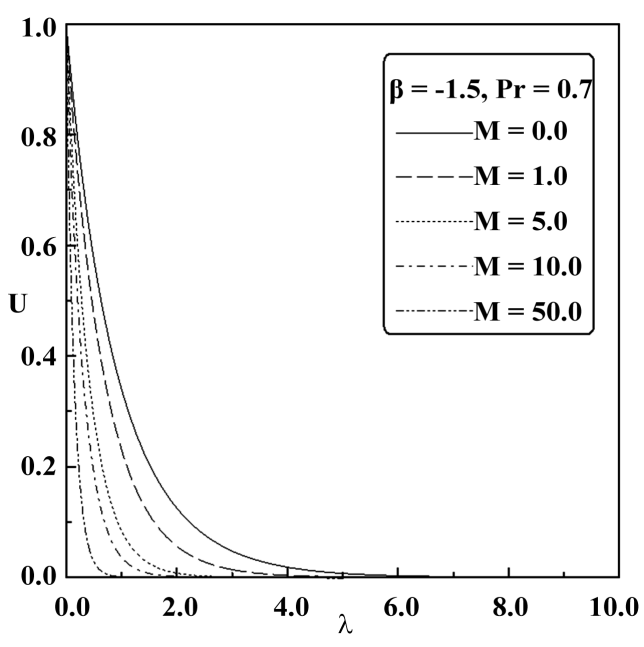

(b)

Figure 3. Horizontal velocity profiles over a range of $M$ with $\operatorname{Pr}=0.7$ for: (a) $\beta=-1.5$; (b) $\beta=1.5$.

\subsection{The Temperature}

Figure 6 illustrates the variation of the temperature profiles $\theta$ for $\beta=1.5$ with Prandtl number $\operatorname{Pr}=0.7$, over a range of $M$. We notice that, the temperature profiles increases as $M$ increases.

Figure 7 describes the distribution of the temperature $\theta$ for $M=0$ with $\operatorname{Pr}=1.0$, over a range of the nonlinear stretching parameter $n$. As seen, with an increase in $n$, the temperature increases.

Figure 8 shows the variation of the temperature profiles $\theta$ for $\beta=1.5$ with $M=0.0$, over a range of the Prandtl number Pr. As seen, the temperature decreases as the Prandtl number increases which consistent with the fact that the thermal boundary layer thickness decreases as the Prandtl number Pr increases.

\subsection{Wall Shear Stress}

The dimensionless wall shear stress $F^{\prime \prime}(0)$ (skin friction) is computed for different values of the Hartmann number $\sqrt{M}$ and the parameter $\beta$. Table 4 shows the numerical values of the skin friction $F^{\prime \prime}(0)$ for different values of the nonlinear stretching parameter $n$ with $M=0.0$. As seen, the absolute value of the dimensionless wall shear stress $\left|F^{\prime \prime}(0)\right|$ increases with increasing $n$, that is because by increasing the values of $n$ 


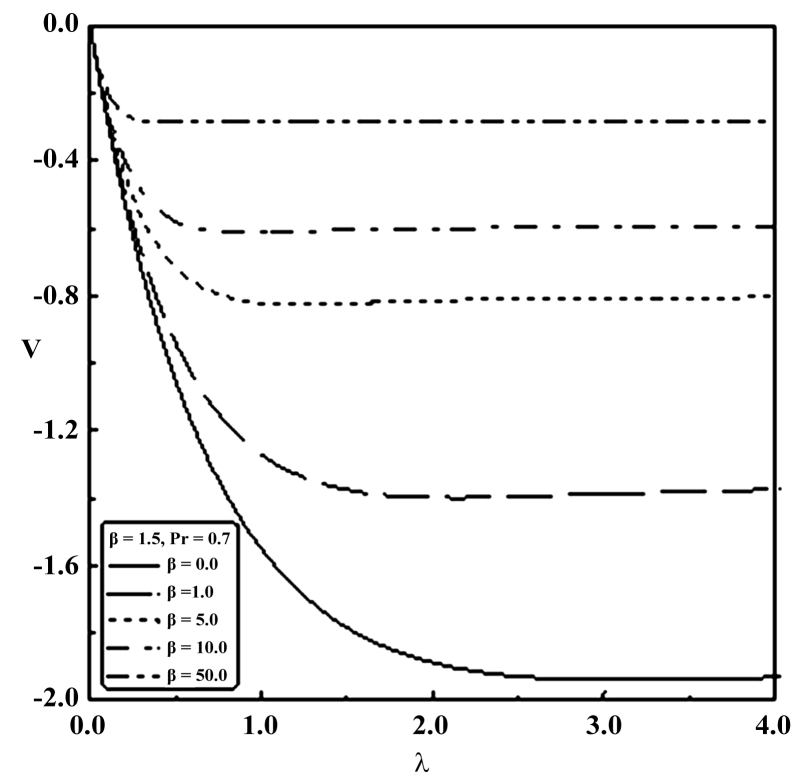

Figure 4. Vertical velocity profiles over a range of $M$ with $\beta=1.5$ and $\operatorname{Pr}=0.7$.

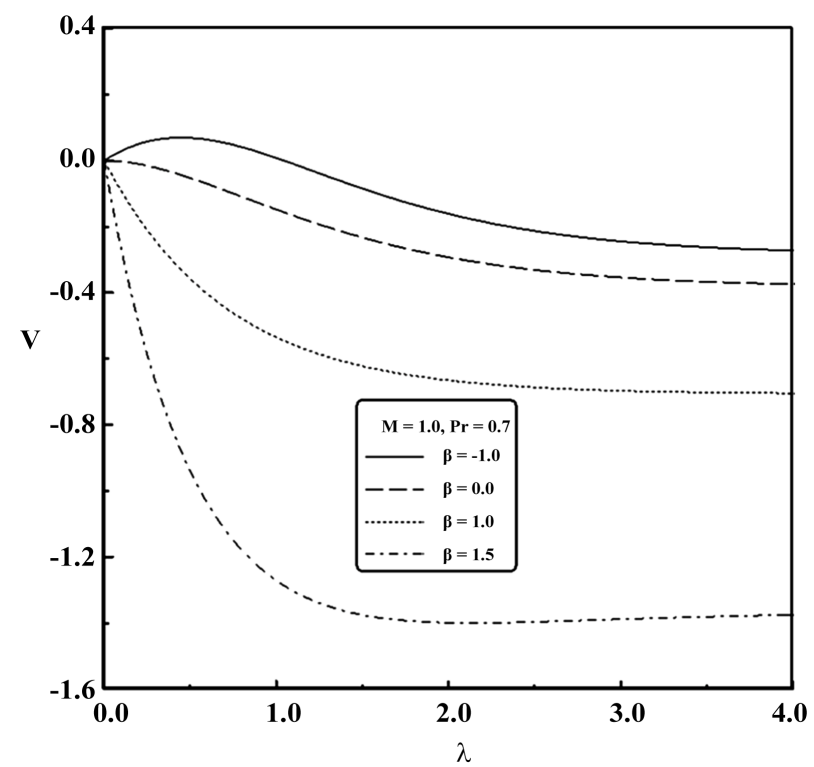

Figure 5. Vertical velocity profiles over a range of $\beta$ with $M=1$ and $\operatorname{Pr}=0.7$.

the layer thickness decreases with an increase in the skin friction at the wall which may cause to lose the smoothness of the stretching wall. So, by increasing the value of $n$, the flow makes the stretching surface rougher. An excellent agreement between our work and other works is absorbed.

Tables 5-8 show the numerical values of $F^{\prime \prime}(0)$ over a range of $M$ with at $\beta=1,1.5,5,-1$ and $\beta=-1.5$, respectively. As $M$ increases, the absolute value of the dimensionless wall shear stress $\left|F^{\prime \prime}(0)\right|$ increases and the thickness of the boundary layer decreases. From Table 8, we noticed that, for small values of $M$ less than 0.4, $\left|F^{\prime \prime}(0)\right|$ decreases as $M$ increases which is consistent with Figure 3(a). Again, an excellent agreement is achieved between our work and other works. No convergent value for $F^{\prime \prime}(0)$ is obtained by Hayat et al. [9] when $\beta=-1.5$ at $M=0.0$, see Table 8 . 


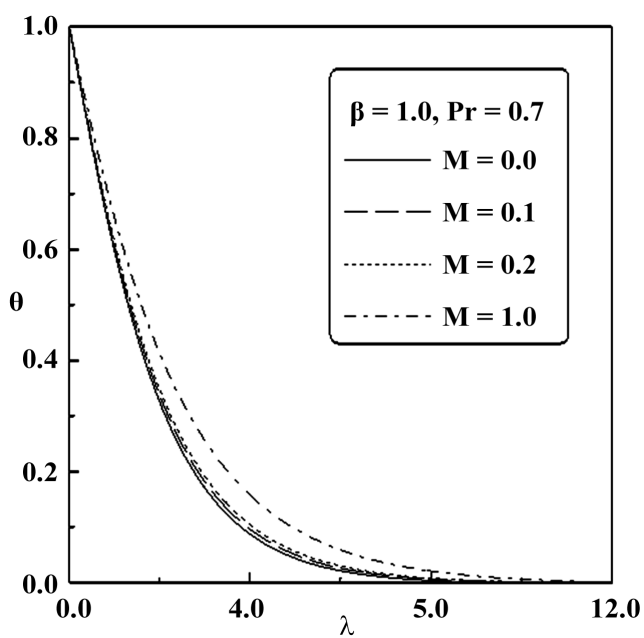

Figure 6. Temperature profiles over a range of $M$ with $\beta=1.5$ and $\operatorname{Pr}=0.7$.

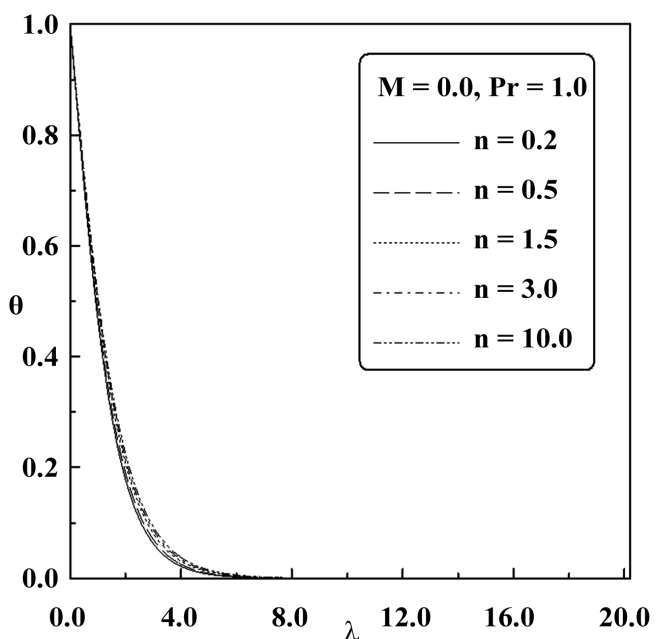

Figure 7. Temperature profiles over a range of $n$ with $M=0.0$ and $\operatorname{Pr}=1.0$.

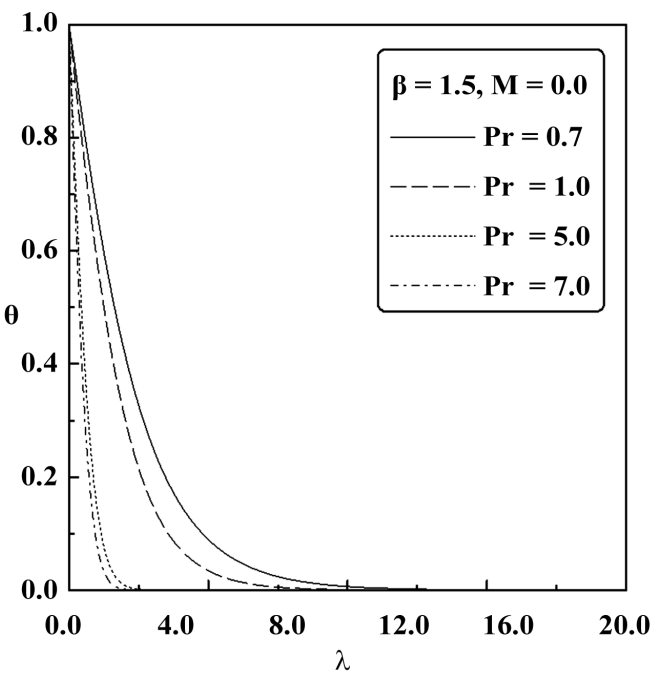

Figure 8. Temperature profiles over a range of $\operatorname{Pr}$ with $\beta=1.5$ and $M=0.0$. 
Table 4. Comparison between the values of $F^{\prime \prime}(0)$ for different $n$ with $M=0.0$.

\begin{tabular}{cccccc}
\hline$n$ & Vajravelu [6] & Cortell [7] & Abbas \& Hayat [8] & Javed et al. [13] & Present work \\
\hline 0.00 & -1.0000 & -0.627547 & -0.627547 & -0.627554 & -0.6275556 \\
0.20 & & -0.766758 & -0.766837 & -0.766837 & -0.7668370 \\
0.50 & -0.889477 & -0.889544 & -0.889543 & -0.8895435 \\
0.75 & -0.953786 & -0.953956 & -0.953956 & -0.9539564 \\
1.00 & -1.000000 & -1.000000 & -1.000000 & -1.0000000 \\
1.50 & -1.061587 & -1.061601 & -1.061601 & -1.0616011 \\
3.00 & -1.148588 & -1.148593 & -1.148593 & -1.1485931 \\
5.00 & & & & -1.1944906 \\
7.00 & -1.1945 & -1.216847 & -1.216851 & -1.216850 & -1.2168503 \\
10.00 & -1.2348 & -1.234875 & -1.234874 & -1.234875 & -1.2348750 \\
20.00 & & -1.257418 & -1.257423 & -1.257423 & -1.2574230 \\
100.00 & & -1.276768 & -1.276773 & -1.276773 & -1.2767731 \\
\hline
\end{tabular}

Table 5. Comparison between the values of $F^{\prime \prime}(0)$ for different $M$ at $\beta=1.0$.

\begin{tabular}{|c|c|c|c|c|c|c|}
\hline$M$ & Pavlov [12] & Ghotbi [10] & Mehmood et al. [11] & Hayat et al. [9] & Fathizadeh et al. [14] & Present work \\
\hline 0 & -1.00000 & & & -1.00000 & -1.00000 & -1.0000000 \\
\hline 1 & -1.41421 & -1.41421 & -1.41421 & -1.41421 & -1.41421 & -1.4142136 \\
\hline 2 & -1.73205 & & -1.73205 & & & -1.7320508 \\
\hline 3 & -2.00000 & & -2.00000 & & & -2.0000000 \\
\hline 4 & -2.23607 & & -2.23607 & & & -2.2360680 \\
\hline 5 & -2.44948 & -2.44948 & -2.44948 & -2.44948 & -2.44948 & -2.4494897 \\
\hline 10 & -3.31662 & -3.31662 & -3.31606 & -3.31662 & -3.31662 & -3.3166248 \\
\hline 15 & -4.00000 & & -4.00100 & & & -4.0000000 \\
\hline 50 & -7.14142 & & & -7.14142 & -7.14142 & -7.1414284 \\
\hline 100 & -10.04987 & & & -10.04987 & -10.0499 & -10.0498756 \\
\hline 500 & -22.38302 & & & -22.38302 & -22.383 & -22.3830293 \\
\hline 1000 & -31.63858 & & & -31.63858 & -31.6386 & -31.6385840 \\
\hline
\end{tabular}

Table 6. Comparison between the values of $F^{\prime \prime}(0)$ for different $M$ at $\beta=1.5$.

\begin{tabular}{cccccc}
\hline$M$ & Chiam [5] & Ghotbi [10] & Hayat et al. [9] & Fathizadeh et al. [14] & Present work \\
\hline 0 & -1.14860 & & -1.1547 & -1.1547 & -1.1486025 \\
1 & -1.52527 & -1.5252 & -1.5252 & -1.5252 & -1.5252751 \\
5 & -2.51615 & -2.5161 & -2.5161 & -2.5161 & -2.5161550 \\
10 & -3.36631 & -3.3663 & -3.3663 & -3.3663 & -3.3663151 \\
50 & -7.16471 & & -7.1647 & -7.1647 & -7.1647100 \\
100 & -10.0664 & & -10.0776 & -10.0776 & -10.0664392 \\
500 & & -22.3904 & -22.3904 & -22.3904733 \\
1000 & & & -31.6438 & -31.6438 & -31.6438511 \\
\hline
\end{tabular}


Table 7. Comparison between the values of $F^{\prime \prime}(0)$ for different $M$ at $\beta=5$.

\begin{tabular}{|c|c|c|c|c|}
\hline$M$ & Chiam [5] & Hayat et al. [9] & Fathizadeh et al. [14] & Present work \\
\hline 0 & -1.90253 & -1.9098 & -1.9098 & -1.9025302 \\
\hline 1 & -2.15290 & -2.1528 & -2.1528 & -2.1529005 \\
\hline 5 & -2.94144 & -2.9414 & -2.9414 & -2.9414400 \\
\hline 10 & -3.69566 & -3.6956 & -3.6956 & -3.6956600 \\
\hline 50 & -7.32561 & -7.3256 & -7.3256 & -7.3256104 \\
\hline 100 & -10.1816 & -10.1816 & -10.1816 & -10.1816304 \\
\hline 500 & & -22.4425 & -22.4425 & -22.4425144 \\
\hline 1000 & & -31.6806 & -31.6806 & -31.6806970 \\
\hline
\end{tabular}

Table 8. Comparison between the values of $F^{\prime \prime}(0)$ for different $M$ at $\beta=-1.0$ and $\beta=-1.5$.

\begin{tabular}{|c|c|c|c|c|c|c|}
\hline \multirow{2}{*}{$M$} & \multicolumn{3}{|c|}{$\beta=-1.0$} & \multicolumn{3}{|c|}{$\beta=-1.5$} \\
\hline & Chiam [5] & Hayat et al. [9] & Present work & Chiam [5] & Hayat et al. [9] & Present work \\
\hline 0 & 0 & 0 & -0.0000010 & 0.72725 & & 0.7272522 \\
\hline 0.1 & -0.13215 & & -0.1321503 & 0.45107 & & 0.4510704 \\
\hline 0.2 & -0.24783 & & -0.2478346 & 0.23038 & & 0.2303800 \\
\hline 0.3 & -0.35006 & & -0.3500590 & 0.05203 & & 0.0520301 \\
\hline 0.4 & -0.44140 & & -0.4414001 & -0.09506 & & -0.0950601 \\
\hline 0.5 & -0.52395 & & -0.5239522 & -0.21922 & & -0.2192231 \\
\hline 1 & -0.85111 & -0.8511 & -0.8511102 & -0.65298 & -0.6532 & -0.6529817 \\
\hline 5 & -2.16287 & -2.1628 & -2.1628674 & -2.08524 & -2.0852 & -2.0852400 \\
\hline 10 & -3.11003 & -3.1100 & -3.1100280 & -3.05623 & -3.0562 & -3.0562320 \\
\hline 50 & & -7.0475 & -7.0475366 & & -7.0238 & -7.0238680 \\
\hline 100 & -9.98335 & -9.9833 & -9.9833469 & -9.96665 & -9.9666 & -9.9666500 \\
\hline 500 & & -22.3532 & -22.3532277 & & -22.3457 & -22.3457703 \\
\hline 1000 & & -31.6175 & -31.6175069 & & -31.6122 & -31.6122354 \\
\hline
\end{tabular}

Table 9. Comparison between the values of $\left(-\theta^{\prime}(0)\right)$ for different values of $\operatorname{Pr}$ and $n$ with $M=0.0$.

\begin{tabular}{ccccccccc}
\hline & \multicolumn{9}{c}{$\operatorname{Pr}=1.0$} & \multicolumn{3}{c}{$\operatorname{Pr}=5.0$} \\
\cline { 2 - 10 }$n$ & $\begin{array}{c}\text { Cortell } \\
{[7]}\end{array}$ & $\begin{array}{c}\text { Abbas \& Hayat } \\
{[8]}\end{array}$ & $\begin{array}{c}\text { Javed et al. } \\
{[13]}\end{array}$ & Present work & $\begin{array}{c}\text { Cortell } \\
{[7]}\end{array}$ & $\begin{array}{c}\text { Abbas \& Hayat } \\
{[8]}\end{array}$ & $\begin{array}{c}\text { Javed et al. } \\
{[13]}\end{array}$ & Present work \\
\hline 0.2 & 0.610262 & 0.610217 & 0.610202 & 0.6102172 & 1.607175 & 1.607925 & 1.607788 & 1.6077882 \\
0.5 & 0.595277 & 0.595201 & 0.595201 & 0.5952010 & 1.586744 & 1.586833 & 1.586783 & 1.5867823 \\
1.5 & 0.574537 & 0.574729 & 0.574730 & 0.5747321 & 1.557463 & 1.557672 & 1.557696 & 1.5576960 \\
3 & 0.564472 & 0.564661 & 0.564662 & 0.5646656 & 1.542337 & 1.542145 & 1.543182 & 1.5431820 \\
10 & 0.554960 & 0.554878 & 0.554879 & 0.5548930 & 1.528573 & 1.528857 & 1.528930 & 1.5289301 \\
\hline
\end{tabular}


Table 10. Comparison between the values of $\left(-\theta^{\prime}(0)\right)$ at $M=0.0$ for different values of $\operatorname{Pr}$ and $n$.

\begin{tabular}{ccccc}
\hline$n$ & \multicolumn{3}{c}{$\operatorname{Pr}=0.71$} & \multicolumn{2}{c}{$\operatorname{Pr}=7.0$} \\
\cline { 2 - 5 } & Vajravelu [6] & Present work & Vajravelu [6] & Present work \\
\hline 1.00 & 0.4590 & 0.4590330 & 1.8953 & 1.8953002 \\
5.00 & 0.4394 & 0.4394328 & 1.8610 & 1.8610243 \\
10.00 & 0.4357 & 0.4357003 & 1.8541 & 1.8541054 \\
\hline
\end{tabular}

Table 11. Numerical values of $\left(-\theta^{\prime}(0)\right)$ for different $M$ at $\beta=-1.0$ and $\operatorname{Pr}=0.7$.

\begin{tabular}{llc}
$M$ & $-\theta^{\prime}(0)$ \\
\hline 0.0 & 0.5644206 \\
0.1 & 0.5454137 \\
0.2 & 0.5280396 \\
0.3 & 0.5124372 \\
0.4 & 0.4983892 \\
0.5 & 0.4856431 \\
1.0 & 0.4416029 \\
5.0 & 0.4039894
\end{tabular}

\subsection{Wall Shear Stress}

Table 9 illustrates the numerical values of the surface heat flux $\left(-\theta^{\prime}(0)\right)$ for different values of the Prandtl number $\operatorname{Pr}$ and nonlinear stretching parameter $n$ with $M=0.0$. The thickness of thermal boundary layer becomes thinner when $\operatorname{Pr}$ increases and this causes an increase in the gradient of the temperature, so, the surface heat flux $\left(-\theta^{\prime}(0)\right)$ increases as $\operatorname{Pr}$ increases. As seen, the results of the present work are in very good agreement with other works, Table 9.

Also, from Table 9, it is noticed that, for fixed value of $\operatorname{Pr}$, the surface heat flux $\left(-\theta^{\prime}(0)\right)$ decreases as nonlinear stretching parameter $n$ increases. Also, the value of $\left(-\theta^{\prime}(0)\right)$ is positive which is consistent with the fact that the heat flows from the sheet surface to the fluid as long as $T_{w}>T_{\infty}$.

Another comparison between the present work with the work of Vajravelu [6] is made, see Table 10.

Table 11 illustrates the numerical values of the surface heat flux $\left(-\theta^{\prime}(0)\right)$ for different values of the $M$ with $\beta=-1.0$ and $\operatorname{Pr}=0.7$. As seen, the surface heat flux $\left(-\theta^{\prime}(0)\right)$ decreases as $M$ increases.

\section{Conclusion}

We have used Lie-group method to obtain the similarity reductions of the MHD boundary-layer equations. By determining the transformation group under which the given system of partial differential equations and its boundary conditions are invariant, we obtained the invariants and the symmetries of these equations. In turn, we used these invariants and symmetries to determine the similarity variables that reduced the number of independent variables. The resulting system of ordinary differential equations was solved numerically using shooting method coupled with Runge-Kutta scheme and the results were plotted. The numerical values of the wall shear stress (skin friction) and surface heat flux were compared with those obtained by other works and they were found in a good agreement.

\section{Acknowledgements}

The author would like to express his appreciations for the potential reviewers for their valuable comments that 
improved the paper and enhanced the results.

\section{References}

[1] Pop, S.R., Grosan, T. and Pop, I. (2004) Radiation Effects on the Flow near the Stagnation Point of a Stretching Sheet. Technische Mechanik, 25, 100-106.

[2] Sakiadis, B.C. (1961) Boundary Layer Behaviour on Continuous Solid Surfaces, II. The Boundary Layer on a Continuous Flat Surface. AIChE Journal, 7, 221-225. http://dx.doi.org/10.1002/aic.690070211

[3] Crane, L.J. (1970) Flow past a Stretching Plate. Journal of Applied Mathematics and Physics (ZAMP), 21, 645-647. http://dx.doi.org/10.1007/BF01587695

[4] Gupta, P.S. and Gupta, A.S. (1977) Heat and Mass Transfer on a Stretching Sheet with Suction or Blowing. Canadian Journal of Chemical Engineering, 55, 744-746. http://dx.doi.org/10.1002/cjce.5450550619

[5] Chaim, T.C. (1995) Hydromagnetic Flow over a Surface Stretching with a Power-Law Velocity. International Journal of Engineering Science, 33, 429-435. http://dx.doi.org/10.1016/0020-7225(94)00066-S

[6] Vajravelu, K. (2001) Viscous Flow over a Nonlinearly Stretching Sheet. Applied Mathematics and Computation, 124, 281-288. http://dx.doi.org/10.1016/S0096-3003(00)00062-X

[7] Cortell, R. (2007) Viscous Flow and Heat Transfer over a Nonlinearly Stretching Sheet. Applied Mathematics and Computation, 184, 864-873. http://dx.doi.org/10.1016/j.amc.2006.06.077

[8] Abbas, Z. and Hayat, T. (2008) Radiation Effects on MHD Flow in a Porous Space. International Journal of Heat and Mass Transfer, 51, 1024-1033. http://dx.doi.org/10.1016/j.ijheatmasstransfer.2007.05.031

[9] Hayat, T., Hussain, Q. and Javed, T. (2009) The Modified Decomposition Method and Padé Approximants for the MHD Flow over a Non-Linear Stretching Sheet. Nonlinear Analysis: Real World Applications, 10, 966-973. http://dx.doi.org/10.1016/j.nonrwa.2007.11.020

[10] Ghotbi, A.R. (2009) Homotopy Analysis Method for Solving the MHD Flow over a Non-Linear Stretching Sheet. Communications in Nonlinear Science and Numerical Simulation, 14, 2653-2663.

[11] Mehmood, A., Munawar, S. and Ali, A. (2010) Comments to: "Homotopy Analysis Method for Solving the MHD Flow over a Non-Linear Stretching Sheet (Commun. Nonlinear Sci. Numer. Simul. 14 (2009) (2653-2663)”. Communications in Nonlinear Science and Numerical Simulation, 15, 4233-4240. http://dx.doi.org/10.1016/j.cnsns.2009.12.039

[12] Pavlov, K.B. (1974) Magnetohydrodynamic Flow of an Incompressible Viscous Fluid Caused by Deformation of a Surface. Magnitnaya Gidrodinamika, 4, 146-147.

[13] Javed, T., Abbas, Z., Sajid, M. and Ali, N. (2011) Heat Transfer Analysis for a Hydromagnetic Viscous Fluid over a Non-Linear Shrinking Sheet. International Journal of Heat and Mass Transfer, 54, 2034-2042. http://dx.doi.org/10.1016/j.ijheatmasstransfer.2010.12.025

[14] Fathizadah, M., Madani, M., Khan, Y., Faraz, N., Yıldırım, A. and Tutkun, S. (2013) An Effective Modification of the Homotopy Perturbation Method for MHD Viscous Flow over a Stretching Sheet. Journal of King Saud UniversityScience, 25, 107-113. http://dx.doi.org/10.1016/j.jksus.2011.08.003

[15] Hill, J.M. (1982) Solution of Differential Equations by Means of One-Parameter Groups. Pitman Publishing Company, Boston.

[16] Seshadri, R. and Na, T.Y. (1985) Group Invariance in Engineering Boundary Value Problems. Springer-Verlag, New York. http://dx.doi.org/10.1007/978-1-4612-5102-6

[17] Olver, P.J. (1986) Applications of Lie Groups to Differential Equations. Springer-Verlag, New York.

[18] Ibragimov, N.H. (1999) Elementary Lie Group Analysis and Ordinary Differential Equations. Wiley, New York.

[19] Boutros, Y.Z., Abd-el-Malek, M.B., Badran, N.A. and Hassan, H.S. (2006) Lie-Group Method for Unsteady Flows in a Semi-Infinite Expanding or Contracting Pipe with Injection or Suction through a Porous Wall. Journal of Computational and Applied Mathematics, 197, 465-494. http://dx.doi.org/10.1016/j.cam.2005.11.031

[20] Boutros, Y.Z., Abd-el-Malek, M.B., Badran, N.A. and Hassan, H.S. (2007) Lie-Group Method of Solution for Steady Two-Dimensional Boundary-Layer Stagnation-Point Flow towards a Heated Stretching Sheet Placed in a Porous Medium. Meccanica, 41, 681-691. http://dx.doi.org/10.1007/s11012-006-9014-X

[21] Boutros, Y.Z., Abd-el-Malek, M.B., Badran, N.A. and Hassan, H.S. (2007) Lie-Group Method Solution for Two-Dimensional Viscous Flow between Slowly Expanding or Contracting Walls with Weak Permeability. Applied Mathematical Modelling, 31, 1092-1108. http://dx.doi.org/10.1016/j.apm.2006.03.026

[22] Abd-el-Malek, M.B., Badran, N.A. and Hassan, H.S. (2007) Lie-Group Method for Predicting Water Content for Immiscible Flow of Two Fluids in a Porous Medium. Applied Mathematical Sciences, 1, 1169-1180. 
[23] Abd-el-Malek, M.B. and Hassan, H.S. (2010) Symmetry Analysis for Steady Boundary-Layer Stagnation-Point Flow of Rivlin-Ericksen Fluid of Second Grade Subject to Suction. Nonlinear Analysis: Modelling and Control, 15, 379-396.

[24] Abd-el-Malek, M.B. and Hassan, H.S. (2010) Solution of Burgers’ Equation with Time-Dependent Kinematic Viscosity via Lie-Group Analysis. Proceedings of the 5th International Workshop "Group Analysis of Differential Equations \& Integrable Systems”, Protaras-Cyprus, 6-10 June 2010, 6-14.

[25] Abd-el-Malek, M.B., Badran, N.A., Hassan, H.S. and Abbas, H.H. (2013) New Solutions for Solving the Problem of Particle Trajectories in Linear Deep-Water Waves via Lie-Group Method. Applied Mathematics and Computation, 219, 11365-11375. http://dx.doi.org/10.1016/j.amc.2013.05.059

[26] Abd-el-Malek, M.B. and Hassan, H.S. (2014) Lie Group Method for Solving the Problem of Fission Product Behavior in Nuclear Fuel. Mathematical Methods in the Applied Sciences, 37, 420-427. http://dx.doi.org/10.1002/mma.2802

[27] Hassan, H.S., Mahrous, S.A., Sharara, A. and Hassan, A. (2014) A Study for MHD Boundary Layer Flow of Variable Viscosity over a Heated Stretching Sheet via Lie-Group Method. Applied Mathematics \& Information Sciences, in Press.

[28] Abd-el-Malek, M.B., Badran, N.A., Hassan, H.S. and Abbas, H.H. (2015) New Solutions for Solving Boussinesq Equation via Potential Symmetries Method. Applied Mathematics and Computation, 251, 225-232. http://dx.doi.org/10.1016/j.amc.2014.11.055

[29] Abd-el-Malek, M.B., Badran, N.A., Hassan, H.S. and Abbas, H.H. (2014) Lie Group Method for Studying the Thermophoresis and Heat Generation Effect on Free-Convection Laminar Boundary-Layer Flow over a Vertical Flat Plate. Submitted for Publication.

[30] Abd-el-Malek, M.B. and Hassan, H.S. (2014) Solution of N-Dimensional Radially Symmetric Non-Linear Diffusion Equation via Symmetry Analysis. Submitted for Publication.

[31] Jacobson, N. (1979) Lie Algebras. Dover, New York.

[32] WafoSoh, C. (2005) Invariant Solutions of the Unidirectional Flow of an Electrically Charged Power-Law Non-Newtonian Fluid over a Flat Plate in Presence of a Transverse Magnetic Field. Communications in Nonlinear Science and Numerical Simulation, 10, 537-548. http://dx.doi.org/10.1016/j.cnsns.2003.12.008 
Scientific Research Publishing (SCIRP) is one of the largest Open Access journal publishers. It is currently publishing more than 200 open access, online, peer-reviewed journals covering a wide range of academic disciplines. SCIRP serves the worldwide academic communities and contributes to the progress and application of science with its publication.

Other selected journals from SCIRP are listed as below. Submit your manuscript to us via either submit@scirp.org or Online Submission Portal.
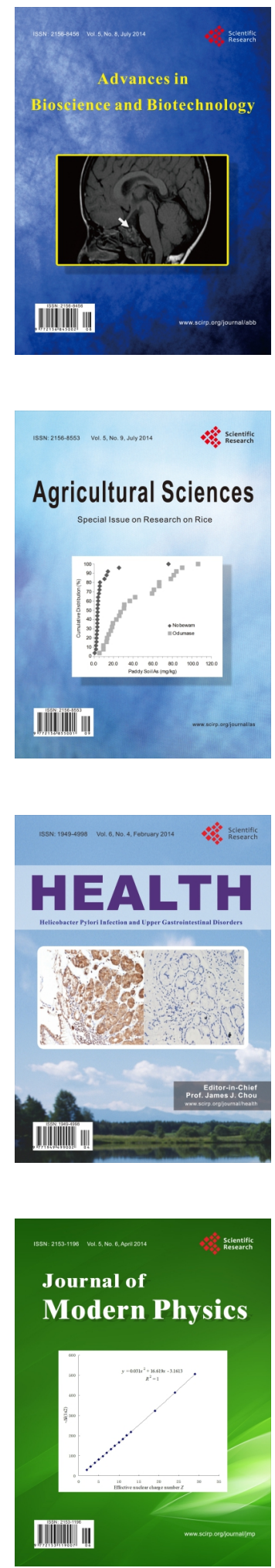
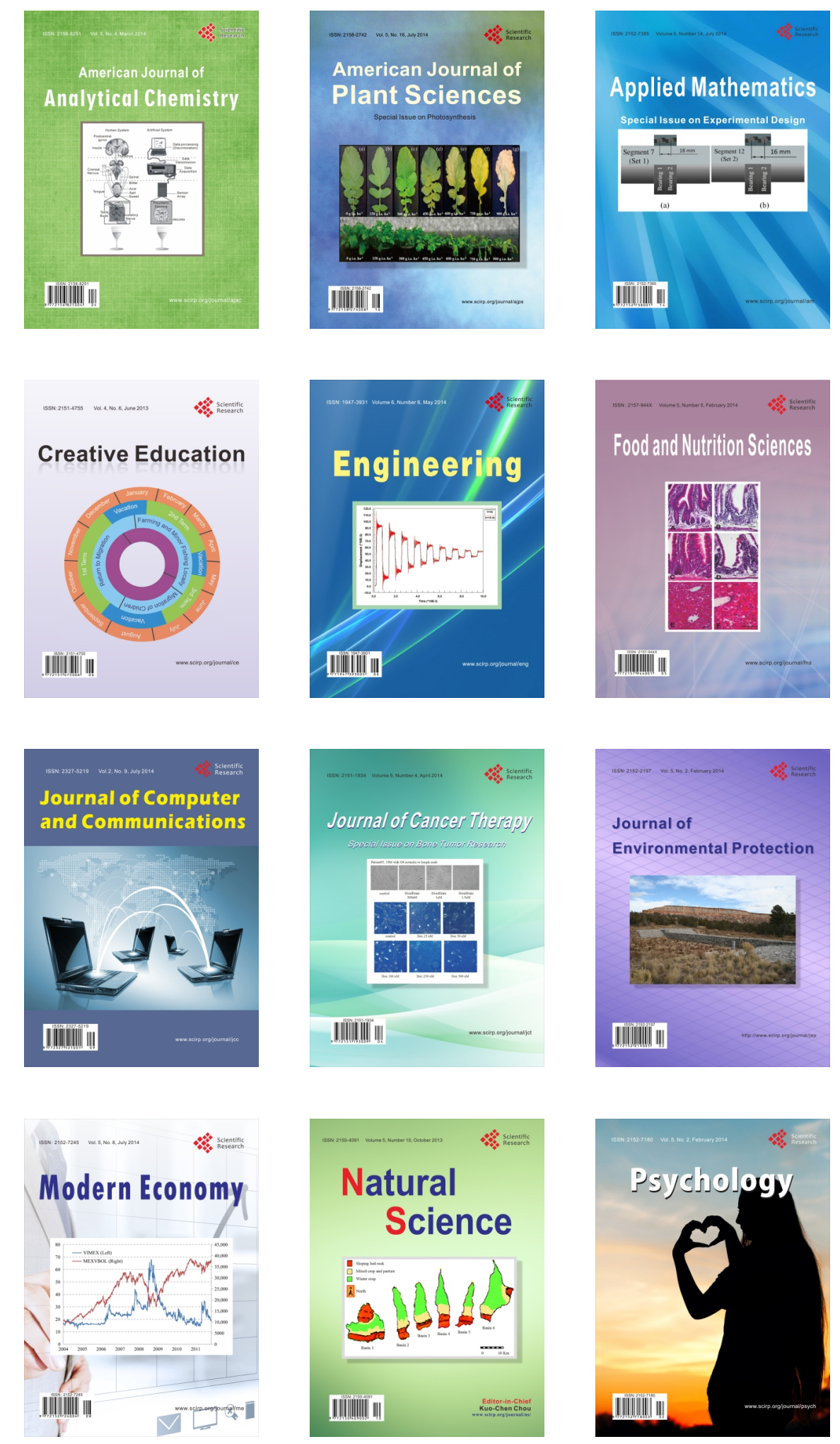